\title{
Educación Matemática y Tecnologías de la Información.
}

\author{
Ricardo López Fernández \\ riclopeusal.es \\ Universidad de Salamanca
}

\section{Introducción al Monográfico}

El desarrollo práctico de los ordenadores y de la propia aplicación de la informática a la educación, desde sus inicios ha estado marcado por sus desarrollos específicos en el ámbito de la enseñanza de las ciencias y particularmente de las matemáticas.

De tal manera que en los últimos años, y a tenor de los recientes análisis teóricos, de las indagaciones empíricas generadas y de las investigaciones experimentales desarrolladas, se ha consolidado la línea que ha revalorizado el papel del computador en la enseñanza en general y de las matemáticas de manera muy específica. Tanto es así, que incluso en el marco de un debate más general pero que sin duda tiene implicaciones directas con la enseñanza de las matemáticas, podemos estar hablando de los inicios de un gran proceso, en el cual la incorporación de las nuevas tecnologías de la información y de la comunicación y sus aplicaciones a la educación puede ser tan profunda que, incluso se convierta en un factor de cambio de los paradigmas clásicos de la educación.

En el campo de la enseñanza de las matemáticas probablemente empecemos a satisfacer las expectativas no cubiertas que, desde los años setenta conjeturaban que el uso de los ordenadores en la educación matemática iba a suponer una revolución, no solo metodológica, de los procesos cognitivos de aprendizaje.

Desde una perspectiva instrumental, las distintas modalidades y tipos en que se pueden clasificar las experiencias desarrollada en la aplicación de las nuevas tecnologías informáticas a la enseñanza de las matemáticas son las siguientes:

El computador tutorial y la enseñanza asistida por ordenador. De amplio desarrollo en la década de los años sesenta y setenta.

El ordenador como herramienta lúdica. Los juegos computacionales.

Cuando la utilización en este campo del ordenador había desarrollado cierto recorrido histórico, se efectuó (Olds,Schwart \& Willie,1980) una subdivisión de esta categoría instrumental atendiendo a la finalidad y objetivos de los diseños de los juegos con ordenador. Así se establecieron por los anteriores autores dos tipos:

$\S \quad$ Los juegos de contenido, cuyo objetivo era, a partir de los juegos propuestos, desarrollar procesos de aprendizaje de tópicos determinados. Los juegos procesuales, cuyo objetivo era el desarrollo de estrategias cognitivas y metacognitivas para la resolución de problemas.

Durante la década de los ochenta este tipo de aplicación educativa, de las nuevas tecnologías, a la enseñanza de las matemáticas se desarrolló ampliamente. Algunos ejemplos que ya están situados en la historia del uso del computador son, Green Globs (Dugdale, 1982) que es una aplicación que permite la representación gráfica de ecuaciones algebraicas ó Guees my Rule ( Barclay, 1985) que tenia como objetivo curricular, el aprendizaje del comportamiento de un conjunto de funciones, a 
partir de la elaboración de tablas de valores numéricos y de sus correspondientes gráficos. Otro ejemplo es el Kings Rules (O’Brien, 1985) que desarrollaba trabajos sobre series en los cuales se planteaba como objetivo, inferir valores de los elementos de una serie a partir de unos primeros datos de la secuencia de elementos.

Aparte de ellos en dicha década se construyeron juegos de estrategias con objetivos que contextualizariamos, dentro del segundo tipo de juegos.

El uso del ordenador en el área de matemáticas, sobre aplicaciones que desarrollaban juegos tenia como fundamento cognitivo, el aportar una herramienta de aprendizaje que potenciara y activara la motivación del alumno. Aunque el estudio del impacto motivacional y afectivo de este tipo de instrumentos es complejo, algunas investigaciones han aportado la evaluación positiva de este impacto (Mandler, 1984) y (Lepper \& Malone, 1987).

Por todo ello, e integrado en las nuevas tecnologías hipermedia, el uso del ordenador para desarrollar juegos matemáticos, bien sobre estrategias de resolución de problemas y toma de decisiones, bien sobre contenidos curriculares específicos, sigue teniendo una amplia vigencia.

Un buen análisis recopilatorio de este tipo de modelo de uso instrumental del juego y del ordenador para la enseñanza de las matemáticas en la década de los ochenta, se puede encontrar en Bright, Harvey \& Wheeeler (1985).

El ordenador como instrumento de simulación.

Los autores anteriores identifican esta función educacional como la característica esencial de la tercera gran clase de aplicaciones informáticas a la enseñanza de las matemáticas. Al respecto, también se distinguen dos tipos de modelos de simulación:

$\S \quad$ Modelo de simulación comparada. Permite, la

$\S \quad$ autoevalución y chequeo que el alumno realiza con la

$\S \quad$ que tiene el sistema.

$\S \quad$ Modelo de simulación sin comparación En donde las

$\S \quad$ posibilidades de chequeo y autoevaluación no son

$\S \quad$ posibles.

En general las simulaciones matemáticas suelen ser del primer tipo.

El ordenador y sus lenguajes de programación como instrumentos enseñanza.

Desde los primeros comienzo con BASIC, hasta el paradigma de esta tipología, el lenguaje LOGO. En general esta modalidad, operó en su momento histórico, sobre la teoría de la transferencia, conjeturando la eficacia del aprendizaje de los lenguajes para desarrollar habilidades y destrezas en la transferencia general de conocimientos. El desarrollo de los lenguajes orientados a objetos, dio al traste con esta estrategia educativa que, por otra parte, tuvo vigencia e impacto durante la década de los ochentas. No obstante, el uso de LOGO, desarrolló el modelo de uso de creación de aplicaciones que desarrollaban "micromundos"

El ordenador como instrumento de desarrollo de aplicaciones de cálculo simbólico

Constituye el modelo de uso más familiar del ordenador: el ordenador como calculadora, el ordenador para el diseño y trabajo con hojas de cálculo, las aplicaciones informáticas para el trabajo 
estadístico, las aplicaciones y usos del ordenador para el cálculo de matrices, o el calculo diferencial e integral, entre otros.

Es evidentemente que este tipo de instrumentos y aplicaciones tienen nombre y apellidos concretos, desde la popular hoja de cálculo EXCEL, hasta programas específicos para el trabajo matemáticos como:

DERIVE (Stoutmyer,1983).

Todos ellos están en la actual década en una amplia fase de aplicación y desarrollo. Para ello se han producido diversas versiones mejoradas y ampliadas de las que se construyeron en los años anteriormente referenciados. Aunque CABRI es situado en el conjunto de aplicaciones de creación de entornos de "micromundos".

Por último, la categoría que referencia al ordenador como instrumento para la creación, desarrollo y uso de la tecnología hipermedia.

El ordenador como instrumento para el desarrollo de aplicaciones y entornos hipermediales cerrados o distribuidos.

Los criterios clasificatorios adoptados, aunque con ciertas modificaciones, han seguido la pauta del trabajo citado de Olds,Schwartz \& Willie. No obstante y como veremos, si se observa las características y posibilidades que para el procesamiento de la información tienen los sistemas hipermedia, y aunque anticipemos el análisis, notaremos como estos posibilitan la integración de todas las categorías de funciones instrumentales de dicho modelo - guía

Esta síntesis de funciones instrumentales opera en la medida en que los sistemas hipermedia, permiten el desarrollo integral de todo esas herramientas y usos. Por ello, los sistemas hipermedia constituyen una plataforma-soporte, como hasta ahora no habíamos tenido, esencial para desarrollar juegos instructivos en cualesquiera de las modalidades que se nos puedan ocurrir, por supuesto, para generar todo tipo de simulaciones con una potencia desconocida hasta esta década, también para implementar aplicaciones para el trabajo y la investigación en los campos de mayor especialización de la matemática, para recrear modelos y estilos cognitivos altamente eficientes y, por último, para posibilitar un trabajo de autoría sobre lenguajes y aplicaciones que, desde la perspectiva de los procesos de aprendizaje, son altamente significativas.

Y ello nos introduce en un ámbito nuevo de estudio e investigación sobre la educación matemática y las TICS. Un ámbito de naturaleza más epistemológica con una amplia proyección sobre los fundamentos en la teoría del aprendizaje de las matemáticas. Así, y desde una perspectiva conectada ya directamente con el estricto ámbito del aprendizaje de las matemáticas, resulta esencial, los estudios sobre aspectos básicos para comprender como aprender matemáticas-y por ello, como enseñar- en base a TIC's.

Desde la perspectiva de las teorías del procesamiento de la información, la capacidad multirepresentacional de los soportes hipermedia, favorece la codificación, la elaboración y la organización del conocimiento. Con ello la capacidad de almacenamiento de este, la eficiencia de su recuperación y la minimización de las interferencias, el decaimiento y el olvido en el proceso de aprendizaje. 
A partir de la formalización modelizada establecida por Golding \& Kaputt (1992) estamos en condiciones de abundar en las argumentaciones sobre la capacidad multi-representacional del soporte hipermedia y su influencia en el procesamiento de la información matemática

En efecto, el soporte hipermedia permite la representación integrada en los tres sistemas dimensionales establecidos en el modelo de Goldin-Kaputt. En algunos de ellos, el sistema de representación soportado en formato multimedia amplía el rango de posibilidades que otros formatos presentaban. En particular, la integración de medios de representación que desarrolla cualquier aplicación multimedia, permite que los nodos de la estructura, soporten conocimiento representado en forma simbólico-notacional, diferentes formas y modelizaciones -, oral - escrita y cualquier tipo de representación figurativa del tipo que hemos llamado "imaginería”.

Esta multi-representación es, hasta el momento, la más completa que en la historia de los formatos y soportes instructivos, hayamos creado. Diferenciándose en cualesquiera de los sistemas de representación de las posibilidades ofertadas y desarrolladas en otros medios como el libro, el diaporama, la televisión ó las aplicaciones informáticas de generaciones previas a los desarrollos multimedia.

Los nodos que creamos con cualesquieras de las aplicaciones multimedia, nos aportan toda una paleta de posibilidades representacionales que nos permiten desarrollar todas las funciones de los distintos sistemas de representación del conocimiento matemático

Otras posibilidades para el desarrollo de este tipo de funciones, por parte del sistema, se pueden establecer a partir de otras de las características del hipermedia, como es su capacidad dinámico interactiva. Los sistemas hipermedia permiten por su disponibilidad, flexibilidad y capacidad dinámica interactiva, adoptar múltiples sistemas notacionales y representacionales enlazados y en interacción continua con el usuario. Aportando, con ello, la integración cognitiva de los conceptos matemáticos, manteniendo así, la significatividad múltiple, propia y asociada de estos. Factor esencial en los procesos eficientes de enseñanza/aprendizaje.

Por todo ello, y en este ámbito del análisis cognitivos de los procesos de enseñanza -aprendizaje de las matemáticas, es central el análisis riguroso de tópicos como los siguientes:

$\S \quad$ La interacción entre los procesos cognitivos y las representaciones del conocimiento matemático.

- Los sistemas hipermedia y la multirepresentación.

$\S \quad$ La representación notacional del pensamiento matemático en los sistemas hipermedia.

- La resolución de problemas, las estrategias de transferencia y los sistemas hipermedia.

- Motivación y sistemas hipermedia.

Investigaciones y contextos de estudio que, por otra parte, tienen importantes proyecciones prescriptivas sobre la teoría del diseño de recursos instructivos en educación matemática.

Las nuevas tecnologías de la comunicación, nos abren nuevas ventanas de representación y transmisión del conocimiento matemático. Previsiblemente, los avances que en la tecnología de la computación y de la comunicación están sucediéndose van a suponer, están suponiendo ya, la posibilidad de creación de nuevos espacios virtuales de representación simbólica que sin duda abren numerosas perspectiva de utilidad didáctica. Y esto por una razón esencial, la significatividad de los sistemas de representación externa del conocimiento matemático facilita, entendiendo estos como productos, su manipulación y por ende su transmisión Esencialmente porque enlazan como hasta ahora ningún soporte de información había conseguido con componentes básicas en la naturaleza del 
pensamiento matemático.

El presente número de la Revista electrónica sobre Teoría de la Educación, nos ha abierto sus puertas para todos aquellos que estamos en el análisis y la reflexión sobre los desarrollos tecnológicos y la educación matemática. Una buena perspectiva de estos ámbitos de investigación se observan a lo largo de las investigaciones y los artículos que componen este número. Agradezco por ello a los editores de la revista que nos hayan dado la oportunidad de este monográfico y a los autores sus contribuciones.

\section{Bibliografía}

BARCLAY, T. (1985). Guess my rule [Software]. Pleasantville, NY: HRM Software.

BRIGHT, G., HARVEY, J., \& WHEELER, M. (1985). Learning and mathematics games (Journal for Research in Mathematics Education Monograph, VoL 1). Reston, VA: National Council of Teachers of Mathematics.

CHAMOSO SÁNCHEZ, J, HERNÁNDEZ ENCINAS, L, LÓPEZ FERNÁNDEZ, R, RODRÍGUEZ SÁNCHEZ, M. (2002) Designing hypermedia tools for solving problem in mathematics.Computer \& Education v. 38,pag 303-317.

COGNITION AND TECHNOLOGY GROUP AT VANDERBILT. (1992a). An anchored instruction approach to cognitive skills acquisition and intelligent tutoring. In J. W. REGION \& V. J. SHUTE (Eds.). Cognitive approaches to automated instruction (pp. 135-170). Hillsdales, NJ: Lawrence Erlbaum Associates.

DUGDALE, S. (1982). Green Globs: A microcomputer application for graphing of equations. Mathematics Teacher, 75, 208-14.

GOLDIN, G. A. (1992a). On developing a unified model for the psychology of mathematical learning and problem solving. In W. GEESLIN \& K. GRAHAM (Eds.), Proceedings of the Sixteenth Annual Meeting of the International Group for the Psychology of Mathematics Education (Vol. 3, pp. 235-261). Durham: University of New Hampshire

KAPUTT, J. (1991a). Notations and representations as mediators of constructive processes. In E. von Glasersfeld (Ed.), Radical constructivism in matbematics education. Dordrecht, Netherlands: Kluwer.

KILPATRICK, J. \& DAVIS, R. B. (1993). Computers and curriculum change in mathematics. In C. KEITEL \& K. RUNVEn (Eds.), Learning from computers: Mathematics education and technology (NATO ASI Series F: Computers and Systems Sciences, Vol. 121, pp. 203-221). Berlin: Springer.

LEPPER, M. R., \& MALONE, T W. (1987). Intrinsic motivation and instructional effectiveness in computer-based education. In R. E. Snow \& M. J. Farr (Eds.), Aptitude, learning and instruction: Conative and affective process analysis (Vol. 3, pp. 255-286). Hillsdale, NJ: Lawrence Erlbaum Associates

LÓPEZ, R. Motivation. Hypermedia Systems and e-learning. In Educational Virtual Spaces in Practices.Ed. Ariel. Barcelona.2005.

MANDLER, G. (1989). Affect and learning: Causes and consequences of emotional interaction. In D. B. McLeod \& V. M. Adams (Eds.), Affect and mathematical problem solving: A new perspective (pp. 3-19). New York: Springer.

NEWELL, A. (1990). Unified theories of cognition. Cambridge, MA: Harvard University Press.. 
OLDS, H., SCHWARTZ, J., \& WILlIE, J. (1980). People and computers: Who teaches whom? Newton, MA: Educational Development Center.

O'BRIEN, T. (1985). The kings rule (Software). Pleasantville, NY: Sunburst Communications..

PEA, R. (1987). Cognitive technologies for mathematics education. In A. Schoenfeld (Ed.), Cognitive science and mathematics education. Hillsdale, NJ: Lawrence Erlbaum Associates.

SPIRO, R. J., FELTOVICH, P. L., JACOBSON, M. J., \& COULSON, R. L. (1991). Cognitive flexibility, construtivism. and hypertext: Random access instruction for advanced knowledge acquisition in ill-structured domains. Educational Technology, 31(5), 24-33.

WOLFRAM, S. (1989). Mathematica. Reading, MA Addison-Wesley.

(C) Ediciones Universidad de Salamanca 